\title{
Perioperative Management of Cardiovascular Implantable Electronic Devices (CIEDs)
}

\author{
Annemarie Thompson · Jacques Prince Neelankavil • \\ Aman Mahajan
}

Published online: 13 July 2013

(C) Springer Science+Business Media New York (outside the USA) 2013

\begin{abstract}
Cardiovascular implantable electronic devices (CIEDs) provide lifesaving support and an improved quality of life to millions of patients. With these patients presenting for elective, urgent, and emergent surgeries, anesthesiologists and other perioperative physicians should become familiar with the various types of devices as well as their perioperative management. The literature regarding the perioperative management of CIEDs has been confusing and at times contradictory; however, more recent publications have offered a thoughtful and educational approach to the perioperative management of CIEDs. This article will summarize some of the current concepts of perioperative management of CIEDs.
\end{abstract}

Keywords Cardiovascular implantable electronic devices · Pacemaker - Internal cardioverter defibrillator . Perioperative management - Electromagnetic interference

A. Thompson $(\bowtie)$

Division of Cardiothoracic Anesthesiology,

Vanderbilt University School of Medicine, 1215 21st Avenue S.,

Suite 5160-MCE NT, Nashville, TN 37232-8274, USA

e-mail: annemarie.thompson@vanderbilt.edu

J. P. Neelankavil

Division of Cardiothoracic Anesthesiology, David Geffen

School of Medicine at UCLA, 757 Westwood Plaza,

Suite 2331L, Los Angeles, CA 9005-7403, USA

e-mail: jneelankavil@mednet.ucla.edu

\section{A. Mahajan}

Department of Anesthesiology, David Geffen School

of Medicine at UCLA, 757 Westwood Plaza, Suite 2331L,

Los Angeles, CA 9005-7403, USA

e-mail: amahajan@mednet.ucla.edu

\section{Introduction}

The rapidly expanding technology of cardiovascular implantable electronic devices (CIEDs) as well as their widespread use not only for bradyarrhythmia and tachyarrhythmia management, but also for congestive heart failure management, has ushered in an era where patients who experience long-term benefit from such therapy are presenting for both cardiac and noncardiac surgery [1, 2]. With approximately one million patients worldwide receiving a pacemaker or implantable cardioverter defibrillator (ICD) every year, patients with CIEDs are a growing population in the perioperative arena [3•]. As "internists of the operating room" who routinely manage complex, life-threatening medical illnesses such as diabetes, anesthesiologists are increasingly asked to address the perioperative management of patients with CIEDs. Management of CIEDs in the perioperative period may be complicated $[4,5]$. Until recently, there was little guidance, confusing literature, and frank misinformation regarding the perioperative management of CIEDs.

The July 2011 Heart Rhythm Society (HRS)/American Society of Anesthesiologists (ASA) Expert Consensus Statement on the perioperative management of patients with CIEDs has sought to inform perioperative physicians and encourage responsible communication between the CIED team that typically manages patients with devices and the perioperative team [6*0]. The HRS/ASA Expert Consensus Statement on the perioperative management of CIEDs has also been endorsed by the AHA, ACC, and STS. Due to its relatively recent publication in the leading electrophysiology journal, most anesthesiologists are not familiar with the document or its details. Additionally, due to the technical complexity of CIEDs, lack of standardization of technology, and the dearth of education regarding these devices in clinical training programs, many advanced 
perioperative physicians find it challenging to learn about the perioperative management of CIEDs.

\section{Perioperative Considerations}

\section{Preoperative Assessment}

Basic terminology/codes for pacemakers and ICDs are reviewed in many manuscripts and texts [7]. Respectively, the pacemaker code represents the chamber paced, the chamber sensed, the response to sensing, and the rate responsive mode.

The need for two-way communication between patient's CIED team that usually takes care of the device programming and function and the operative team was emphasized by the Heart Rhythm Society (HRS)/American Society of Anesthesiologists (ASA) Expert Consensus Statement published in July 2011. The most effective means of caring for the patient with a CIED will occur when the CIED team that usually manages the patient's device is given the necessary details of the operative procedure in order to create an individualized, perioperative prescription. The information that should be exchanged is summarized in Tables 1 and 2 .

\section{Electromagnetic Interference}

Electromagnetic interference (EMI) in the preoperative settings can interfere with the functioning of both pacemakers and ICDs, presenting a challenge to the management of these devices [8-10]. Although there are several potential sources of EMI, the major source is monopolar electrocautery, particularly if the source of EMI is within 6 inches $(15 \mathrm{~cm})$ of the generator. Bipolar electrocautery is usually not a concern, as the electrical current field is small, limited to the two poles at the end of the stylus [11].

Table 1 Essential information to be given to the CIED team by the perioperative team

Type of procedure

Anatomic location of procedure

Patient position during procedure

If and where monopolar electrocautery will be used

Any other sources of EMI

Any planned cardioversion or defibrillation

Surgical venue (operating room, office-based procedure, etc.)

Postprocedural plan (hospital admission, discharge within $12 \mathrm{~h}$, etc.)

Unusual circumstances (surgery encroaching upon device leads or generator, etc.)

$E M I$ electromagnetic interference
Table 2 Essential elements to be communicated to the perioperative team by the CIED team

Date of last device interrogation-recommend within 6 months for ICD or cardiac resynchronization therapy (CRT) device, 12 months for pacemaker

Device type, manufacturer, and model

Indication for device placement

Battery longevity

Any leads placed within the last 3 months

Current programming

Is the patient pacemaker-dependent?

Device response to magnet placement

Any alert status on device? (such as manufacturing issues)

Last pacing threshold

Individualized perioperative recommendation/prescription based on patient information, device characteristics, and surgical factors

ICD implantable cardioverter defibrillator

Monopolar electrocautery is the most frequently used type of electrocautery because it has both cutting as well as coagulation capabilities. Modern CIEDs have evolved to produce better shielding from EMI. Contrary to common belief, shielding from EMI does not reduce the potential to induce a "reset" mode (which is discussed later), but rather shielding reduces the potential to induce oversensing in the CIED. Oversensing in a pacemaker means the pacemaker "sees" the EMI generated artifacts as intrinsic cardiac electrical activity and, therefore, does not initiate a paced rhythm. Such failure to initiate a paced rhythm (inhibition of the pacemaker) can compromise hemodynamics in a pacemaker-dependent patient. If oversensing occurs in an ICD, EMI may be misinterpreted by the ICD as a malignant tachyarrhythmia, which may cause the patient to receive an inappropriate shock [12]. EMI can also cause inappropriate pacing in patients with rate responsive pacemakers. Rate responsiveness is a feature that allows the pacemaker to increase its rate secondary to perceived physical exertion. Common rate responsive technology uses minute ventilation sensors, which can be triggered by EMI, resulting in inappropriate increases in pacing rate [13, 14].

In contrast to the ASA Practice Advisory [15], the HRS/ ASA Consensus Statement suggests that due to the decreased likelihood of EMI related interference seen when surgery is below the umbilicus, the patient should proceed to surgery with no magnet application to the device or reprogramming. However, there should be a magnet available with the magnet function of the device known in case of a change in surgical plan or unexpected EMI. Alternatively, a magnet can be applied if perioperative physicians feel more comfortable with that approach, provided the perioperative team is aware of the magnet function for that particular device. 
Magnets

Interestingly, magnets were never developed for perioperative use, although they are now widely considered for shielding of EMI or treatment of CIED emergencies. Magnets were initially used to determine battery life in CIEDs. However, both magnet application and reprogramming are options for eliminating EMI. The choice of reprogramming versus magnet application largely depends on the type of surgery, patient position, accessibility of programmer, and knowledge of magnet function of a particular patient's device. It should be emphasized that a magnet has different effects when applied to a pacemaker or to an ICD. In the majority of pacemakers, magnet application induces an asynchronous mode. Magnet application to an ICD inhibits tachyarrhythmia detection, thus preventing therapy, i.e., delivery of shocks. However it does NOT change the mode of the underlying pacemaker. Therefore, a magnet placed over an ICD will not induce asynchronous mode in the underlying pacemaker. For patients with an ICD who are pacemakerdependent, reprogramming is the preferred option if EMI is a significant concern.

For a few ICDs, notably an older model known as the PRIZM series (Boston Scientific-Guidant), the magnet mode can be changed to induce suspension of tachyarrhythmia therapy only after the magnet is applied for $30 \mathrm{~s}$. Tachytherapy will be reactivated when the magnet is reapplied for $30 \mathrm{~s}$. For any device, the magnet response should be confirmed with the CIED team prior to magnet application. While most pacemakers will convert to asynchronous mode when a magnet is applied to the generator, some devices have a programmable magnet function that can prevent conversion to asynchronous mode when a magnet is placed over the generator. For this reason, it is important to consult with the patient's CIED team to determine a particular device's magnetic response.

\section{Electrical Reset}

Pacemakers and ICDs are reliable devices that may malfunction [16, 17]. A type of malfunction that is commonly misunderstood is the concept of electrical reset. In the perioperative setting, this is thought to very rarely occur when an energy surge directly contacts the pulse generator, resulting in a major hardware/software failure. The reset mode is unique to each manufacturer and serves as a safety backup in the case of catastrophic failure. The most common cause of electrical reset is therapeutic radiation, not electrocautery or external cardioversion/defibrillation [18-20]. Neither magnet application nor reprogramming will prevent electrical reset. The best prevention of electrical reset is to direct energy away from the pulse generator $(>15 \mathrm{~cm})$ and to place the dispersive electrode ("Bovie pad") in proper position to prevent current flow across the generator.
Fig. 1 Suggested sample algorithm to assist in the routine management of CIEDs. The action items outlined in red indicate instances where either a magnet or reprogramming may be necessary in the routine management of the CIED. CIED cardiovascular implantable electronic devices, $E M I$ electromagnetic interference, ICD implantable cardioverter defibrillator, $C R T$ cardiac resynchronization therapy

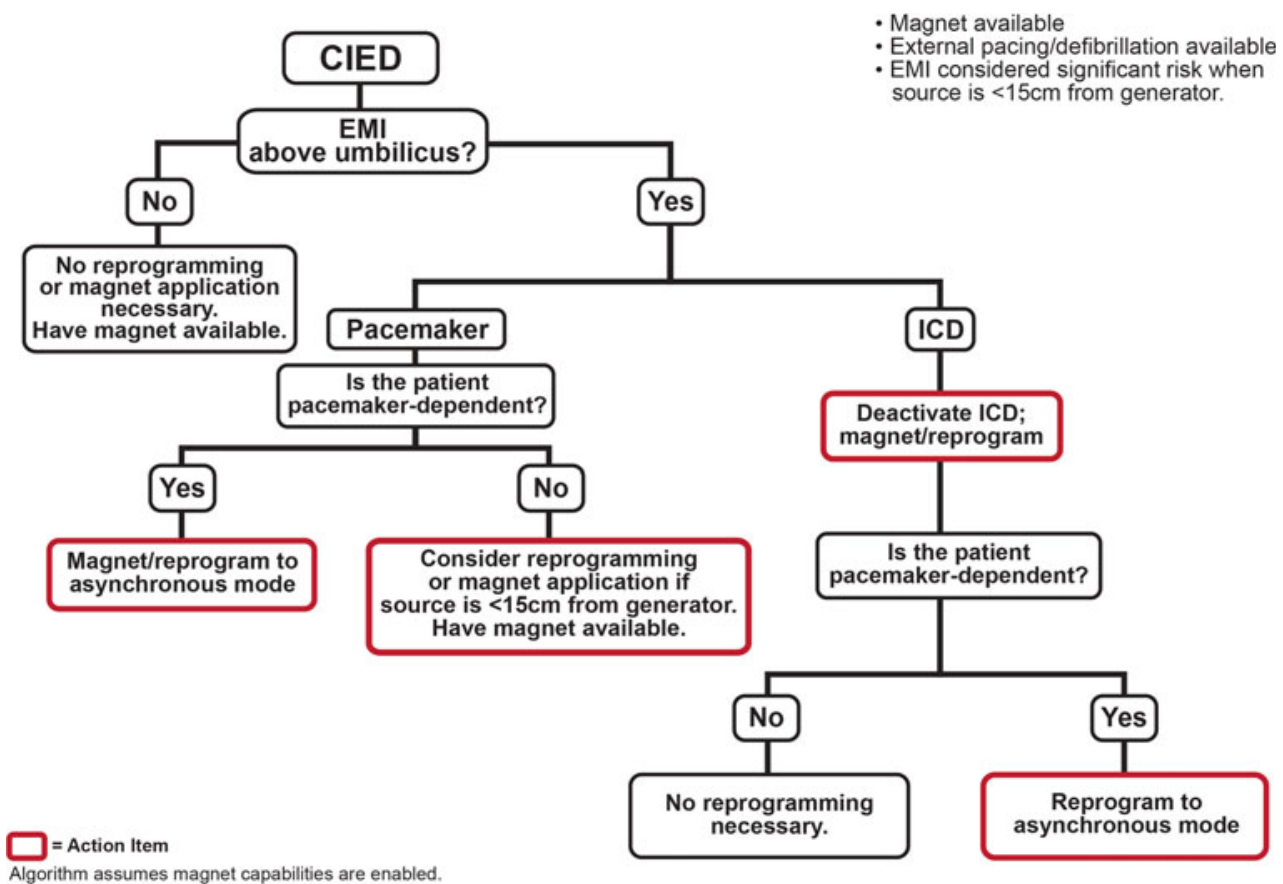

Algorithm assumes magnet capabilities are enabled. Consider consulting CIED team for CRT devices. 
Perioperative Algorithm for Patients with CIEDs Presenting for Non-U'rgent Surgery

Although current recommendations strongly recommend a tailored approach to perioperative CIED management based on the patient's CIED history, type of surgery, and medical co-morbidities, it can be helpful to have a general idea of the management of CIEDs when patients present for surgery. There are numerous sources available that suggest approaches to perioperative management [21-23]; a sample algorithm is shown in Fig. 1.

For patients who have had a CIED placed within the prior six weeks, care must be taken during invasive procedures such as central line insertion and pulmonary artery catheter placement, as the risk for dislodgment is increased until the pacing/ICD leads become solidly fixated within the endocardium. Additionally, patients with a cardiac resynchronization device (also called a biventricular pacemaker or biventricular ICD) have a coronary sinus lead that can be more easily dislodged when compared to a chamber lead since there is no mechanism of lead fixation in the coronary sinus.

\section{Emergency Protocol}

Patients with CIEDs may present for urgent/emergent surgery, leaving little or no time for device interrogation or CIED team consultation. In such instances, identification of the device is important. If the patient does not know the type of device (pacemaker, ICD, biventricular pacemaker, biventricular ICD, etc.), chest radiography can be used to help identify the device.

An electrocardiogram or rhythm strip should be examined for pacing spikes. If pacing spikes are noted before most or all of either the $\mathrm{P}$ wave or the QRS complexes, then the patient should be treated as if he/she is pacemakerdependent. All pacemaker-dependent patients and patients with ICDs should have transcutaneous pacing and defibrillator pads placed during emergency cases. A magnet should always be available and its use is recommended in the pacemaker-dependent patient as well as the patient with an ICD. Regardless of the type of device, interrogation of the CIED under the direction of a physician knowledgeable in the function of devices should occur as soon as possible. The emergency protocol is described in the HRS/ASA Expert Consensus Statement.

\section{Postoperative Management of CIEDs}

Postoperative management of CIEDs should be addressed in the preoperative assessment, but may need modifications based on intraoperative events. As with the preoperative assessment, the plan for perioperative care should be individualized to meet the needs of the patient. The patient's medical history, history of adherence to medical recommendations, type of device and surgical course will help determine the most appropriate postoperative management. Recommendations from the HRS/ASA Expert Consensus Statement suggest that patients receive either a telephonic or clinical evaluation of their device within 30 days of surgery, except in a few circumstances. Devices will require interrogation in the immediate postoperative period in only a few instances. First, if any programming changes were made preoperatively, then the device should be reprogrammed to its preoperative settings prior to discharge from a telemetry unit. If there is a concern for nonadherence to recommended follow-up within 30 days, then the device should be interrogated in the immediate postoperative period. Lastly, if there are unusual circumstances regarding the surgery, such as large blood loss/fluid administration, intraoperative defibrillation/cardioversion, or concern for direct damage to the CIED, then the device should be interrogated in the immediate postoperative period.

\section{Conclusions}

Patients with CIEDs are presenting for elective surgery in ever increasing numbers. Anesthesiologists will increasingly be called upon to assist in the development of institution-specific strategies based on local resources to efficiently manage patients with CIEDs who present for surgery. A basic understanding of CIEDs combined with continuing education about the perioperative management of these devices will assist anesthesiologists in the important task of caring for patients with CIEDs.

Disclosure Annemarie Thompson, Jacques Prince Neelankavil, and Aman Mahajan declare that they have no conflict of interest.

Compliance with ethical requirements This article does not contain any studies with human or animal subjects performed by any of the authors.

\section{References}

Papers of particular interest, published recently, have been highlighted as:

- Of importance

- Of major importance

1. Moss AJ, Zareba W, Hall WJ, et al. Prophylactic implantation of a defibrillator in patients with myocardial infarction and reduced ejection fraction. N Engl J Med. 2002;346:877-83.

2. Cleland JG, Daubert JC, Erdmann E, Freemantle N, Gras D, Kappenberger L, Tavazzi L, Cardiac-Heart Failure (CARE-HF) 
Study Investigators. The effect of cardiac resynchronization on morbidity and mortality in heart failure. $\mathrm{N}$ Engl $\mathrm{J}$ Med. 2005;352(15):1539-49.

3. - Greenspon AJ, Patel JD, Lau E, Ochoa JA, Frisch DR, Ho RT, Pavri BB, Kurtz SM. Trends in permanent pacemaker implantation in the United States from 1993 to 2009: increasing complexity of patients and procedures. J Am Coll Cardiol. 2012;16:60(16):1540-5. doi:10.1016/j.jacc.2012.07.017. This article describes important trends in pacemaker implantation in the United States. The majority of pacemakers inserted are dual chamber pacemakers regardless of the indication for insertion.

4. Amin MS, Ellenbogen KA. Focus on management of pacemaker and ICD advisories, recalls, and alerts. Curr Treat Options Cardiovasc Med. 2006;8:347-52.

5. Wilkoff BL. Pacemaker and ICD malfunction-an incomplete picture. JAMA. 2006;295:1944-6.

6. • Crossley GH, Poole JE, Rozner MA, Asirvatham SJ, Cheng A, Chung MK, Ferguson TB Jr, Gallagher JD, Gold MR, Hoyt RH, Irefin S, Kusumoto FM, Moorman LP, Thompson A. The Heart Rhythm Society (HRS)/American Society of Anesthesiologists (ASA) Expert Consensus Statement on the perioperative management of patients with implantable defibrillators, pacemakers and arrhythmia monitors: facilities and patient management this document was developed as a joint project with the American Society of Anesthesiologists (ASA), and in collaboration with the American Heart Association (AHA), and the Society of Thoracic Surgeons (STS). Heart Rhythm 2011;8:1114-54. A consensus statement on the perioperative management of CIEDs developed by a multidisciplinary writing group. This consensus statement includes an extensive tabular review of the literature and practical clinical recommendations for management.

7. Bernstein AD, Daubert JC, Fletcher RD, Hayes DL, Lüderitz B, Reynolds DW, Schoenfeld MH, Sutton R. The revised NASPE/ BPEG generic code for antibradycardia, adaptive-rate, and multisite pacing. North American Society of Pacing and Electrophysiology/British Pacing and Electrophysiology Group. Pacing Clin Electrophysiol. 2002;25(2):260-4.

8. Belott PH, Sands S, Warren J. Resetting of DDD pacemakers due to EMI. Pacing Clin Electrophysiol. 1984;7:169-72.

9. Godin JF, Petitot JC. STIMAREC report. Pacemaker failures due to electrocautery and external electric shock. Pacing Clin Electrophysiol. 1989;12:1011.

10. Mangar D, Atlas GM, Kane PB. Electrocautery-induced pacemaker malfunction during surgery. Br J Anaesth. 1991;38:616-8.
11. Lee D, Sharp VJ, Konety BR. Use of bipolar power source for transurethral resection of bladder tumor in patient with implanted pacemaker. Urology. 2005;66:194.

12. Casavant D, Haffajee C, Stevens S, Pacetti P. Aborted implantable cardioverter defibrillator shock during facial electrosurgery. Pacing Clin Electrophysiol. 1998;21(6):1325-6.

13. Van Hemel NM, Hamerlijnck RP, Pronk KJ, et al. Upper limit ventricular stimulation in respiratory rate responsive pacing due to electrocautery. PACE. 1989;12:1720-3.

14. Wong DT, Middleton W. Electrocautery-induced tachycardia in rate-adaptive pacemaker. Anesthesiology. 2001;94:710-1.

15. American Society of Anesthesiologists. Practice advisory for the perioperative management of patients with cardiac implantable electronic devices: pacemakers and implantable cardioverterdefibrillators: an updated report by the American society of anesthesiologists task force on perioperative management of patients with cardiac implantable electronic devices. Anesthesiology. 2011;114:247-61.

16. Maisel WH. Pacemaker and ICD generator reliability: metaanalysis of device registries. JAMA. 2006;295:1929-34.

17. Maisel WH, Moynahan M, Zuckerman BD, et al. Pacemaker and ICD generator malfunctions: analysis of Food and Drug Administration annual reports. JAMA. 2006;295:1901-6.

18. Furman S, Fisher JD. Endless loop tachycardia in an AV universal [DDD] pacemaker. Pacing Clin Electrophysiol. 1982;5: 486-9.

19. Katzenberg CA, Marcus FI, Heusinkveld RS, Mammana RB. Pacemaker failure due to radiation therapy. Pacing Clin Electrophysiol. 1982;5:156-9.

20. Rozner M. Pacemaker misinformation in the perioperative period: programming around the problem [comment]. Anesth Analg. 2004;99:1582-4.

21. Pinski SL, Trohman RG. Interference in implanted cardiac devices, part II. Pacing Clin Electrophysiol. 2002;25(10):1496509.

22. Mahlow WJ, Craft RM, Misulia NL, et al. A perioperative management algorithm for cardiac rhythm management devices: the PACED-OP protocol. Pacing Clin Electrophysiol. 2013;36: 238-48.

23. Goldschlager N, Epstein A, Friedman P, et al. Environmental and drug effects on patients with pacemakers and implantable cardioverter/defibrillators: a practical guide to patient treatment. Arch Intern Med. 2001;161:649-55. 